\title{
Personnel Evaluation Under Intuitionistic Fuzzy Environment
}

\author{
Melda KOKOC*1, Suleyman ERSOZ ${ }^{2}$
}

\begin{abstract}
Submitted: 07/05/2020 Accepted: 29/06/2020
Abstract: In this paper, a new approach is proposed to solve multi-person and multi-attribute evaluation problems under an intuitionistic fuzzy environment. The proposed evaluation approach is mainly grounded on the integration of the score function and aggregation operator for intuitionistic fuzzy sets. To illustrate the application of the novel approach, a numerical example for evaluating engineers according to attributes of T-shaped engineers is given. The novelty of this study is that it defines T-shaped engineer selection as a multiattribute evaluation problem in the literature for the first time. In addition, it proposes an integrated intuitionistic fuzzy evaluation approach in which the candidates are evaluated at both technical (hard) skills and non-technical (soft) skills. This study contributes to the literature as it provides a novel insight into the theoretical ground of the personnel selection problem.
\end{abstract}

Keywords: intuitionistic fuzzy set, multi-attribute, multi-person, personnel selection, T-shaped engineer

\section{Introduction}

An intuitionistic fuzzy set (IFS) introduced by Atanassov [1,2] is a powerful tool to deal with vagueness. An important characteristic of IFS is that it assigns to each element a membership degree and a non-membership degree. Thus, the IFSs form an extension of Zadeh [3]'s fuzzy set that only assigns a membership degree to each element. Many authors have paid attention to the IFS theory. This theory has been applied many areas such as; data envelopment analysis [4], medical diagnosis [5-7], minimum cost flow problem [8], multi-attribute evaluation [9-14], pattern recognition [15-17], information fusion [18-19], service quality assessment [20-22].

Multi-person and multi-attribute evaluation, which was first introduced in the early 1970s, includes a common human activity, which involves the evaluators to participate in the assessment process in order to find the most suitable alternative considering the weights of factors and sub-factors. In this activity, the information about attribute values is usually uncertain or fuzzy owing to the vagueness of the inherently subjective nature of human thinking [23]. The personnel selection for new positions is one of the much-discussed research areas in the multi-person and multi-attribute evaluation, and a great number of researches have been conducted in this area [24-28].

The demand for young professionals who have both a depth of knowledge in one system and the ability to perform crossdisciplinary collaboration gradually comes into prominence for the personnel selection process in the 21 st century [29]. The Tshaped engineers called as new employees of the digital age have responded to this demand [30]. In other words, the horizontal bar of the ' $\mathrm{T}$ ' represents a breadth of expertise, ability to engage with other experts across a variety of systems and disciplines; the

\footnotetext{
${ }^{1}$ Education and External Relations Coordination Office, Gazi University, Ankara-06560, TURKEY

ORCID ID: 0000-0003-2035-9777

${ }^{2}$ Industrial Eng., Kirlkkale University, Kirlkkale-71450, TURKEY

ORCID ID: 0000-0002-7534-6837

${ }^{*}$ Corresponding Author: Email:meldakokoc@gazi.edu.tr
}

vertical bar of the ' $\mathrm{T}$ ' represents a depth of expertise in a specific knowledge domain [29,31].

Despite the increasing interest and demand for $\mathrm{T}$-shaped engineers, there are not enough studies in the literature. Although there are studies investigating the skills that T-shaped engineers should acquire for 21st-century conditions [32-49], there are no studies evaluating the engineers' skills within the framework of the T-shaped engineer.

In this study, a new approach is proposed to solve multi-person and multi-attribute evaluation problems in an intuitionistic fuzzy environment for a new application area related to personnel selection. The highlights of the proposed approach and its contributions to the literature are as follows:

(1) It can be used in cases where the weights of factors and subfactors may vary depending on the field of application.

(2) It is the first multi-attribute evaluation approach that is to consider the opinions of expert groups rather than experts.

(3) It reduces losing or distorting the assessment information in the process of aggregation to the minimum, as it uses the entropy to convert the aggregated assessment of groups into weights.

(4) It helps fill research gaps in this field by presenting an evaluation approach for T-shaped engineering skills.

(5) In similar studies [26, 50-52] it isn't explained which characteristics are used for determining the importance level of the experts are not explained. In this study, experts are grouped considering specific characteristics, and then, importance levels and IFNs are defined for these groups.

The rest of this paper is structured as follows. In section 2 the preliminaries related to intuitionistic fuzzy sets are presented to facilitate understanding of the approach. The proposed approach is introduced in section 3, and then, an illustrative example is presented related to personnel selection in section 4. At the beginning of the illustrative example, the technical and nontechnical skills, which must be acquired by engineers to become T-shaped, are determined by literature research and analysis of job advertisements. These skills are divided into factors and subfactors through literature research and expert opinions. After the 
weights of factors and sub-factors are determined by evaluating the opinions of the experts, the proposed approach for the selection of engineers is carried out.

\section{Preliminaries}

In this section, some basic concepts related to intuitionistic fuzzy sets and intuitionistic fuzzy numbers are reviewed in order to facilitate further discussions.

Definition 2.1. [1] Let $X$ be a universe of discourse, then an intuitionistic fuzzy set $\tilde{A}$ is defined as $\tilde{A}=\left\{\left\langle x, \mu_{\tilde{A}}(x), v_{\tilde{A}}(x)\right\rangle \mid x \in X\right\}$ where $\mu_{\tilde{A}}: X \rightarrow[0,1] \quad$ and $v_{\tilde{A}}: X \rightarrow[0,1]$ under the condition $0 \leq \mu_{\tilde{A}}(x)+v_{\tilde{A}}(x) \leq 1 . \mathrm{Xu}$ (2007) defined IFS as an ordered pair $\tilde{A}=\left(\mu_{\tilde{A}}(x), v_{\tilde{A}}(x)\right)$ for convenience. $\mu_{\tilde{A}}(x)$ and $v_{\tilde{A}}(x)$ represent the degrees of membership and non-membership of the element $x$ to the set $\tilde{A}$, respectively. Furthermore, IFSs reduce to a crisp set when the value of $\mu_{\tilde{A}}(x)=1-v_{\tilde{A}}(x)$ is equal to 0 or 1 .

Definition 2.2. [1] Hesitation degree of the intuitionistic fuzzy set $\tilde{A}$ is referred as $\pi_{\tilde{A}}(x)=1-\left(\mu_{\dot{A}}(x)+v_{\tilde{A}}(x)\right), x \in X$. Also, there is $0 \leq \pi_{\hat{A}}(x) \leq 1$ for $\forall x \in X$. If $\mu_{\dot{A}}(x)$ and $v_{\dot{A}}(x)$ are both continuous functions, distance between each pair of functions means the hesitation part of $x$ to the set $\tilde{A}$. It is clear that the value of element $x$ of set $\tilde{A}$ is more uncertain when the value of $\pi_{\AA}(x)$ is large and more certain when the value of $\pi_{\AA}(x)$ is small.

Definition 2.3. $[1,53]$ Assume $\tilde{A}=\left(\mu_{\tilde{A}}(x), v_{\tilde{A}}(x)\right)$ and

$\tilde{B}=\left(\mu_{\tilde{B}}(x), v_{\tilde{B}}(x)\right)$ are IFNs.

(1) (Complement) $\overline{\widetilde{A}}=\left(v_{\ddot{A}}(x), \mu_{\tilde{A}}(x)\right)$

(2) (Intersection) $\tilde{A} \cap \tilde{B}=\min \left\{\mu_{\tilde{A}}(x), \mu_{\tilde{B}}(x)\right\}, \max \left\{v_{\tilde{A}}(x), v_{\tilde{B}}(x)\right\}$

(3) (Union) $\tilde{A} \cup \tilde{B}=\max \left\{\mu_{\dot{A}}(x), \mu_{\tilde{B}}(x)\right\}, \min \left\{v_{\tilde{A}}(x), v_{\tilde{B}}(x)\right\}$

(4) (Sum) $\tilde{A} \oplus \tilde{B}=\left(\mu_{\tilde{A}}(x)+\mu_{\tilde{B}}(x)-\mu_{\tilde{A}}(x) \mu_{\tilde{B}}(x), v_{\tilde{A}}(x) v_{\tilde{B}}(x)\right)$

(5) (Product) $\tilde{A} \otimes \tilde{B}=\left(\mu_{\bar{A}}(x) \mu_{\tilde{B}}(x), v_{\bar{A}}(x)+v_{\tilde{B}}(x)-v_{\tilde{A}}(x) v_{\tilde{B}}(x)\right)$

(6) (Scale Multiplication) $\delta \tilde{A}=\left(1-\left(1-\mu_{\tilde{A}}(x)\right)^{\delta}, v_{\tilde{A}}(x)^{\delta}\right), \delta>0$

(7) (Power) $\tilde{A}^{\delta}=\left(\mu_{\tilde{A}}(x)^{\delta}, 1-\left(1-v_{\tilde{A}}(x)\right)^{\delta}\right), \delta>0$

Definition 2.4. [54] The score function $S$ defined as the difference and the sum of the membership function $\left(\mu_{\tilde{A}}(x)\right)$ and the non-membership function $\left(v_{\tilde{A}}(x)\right)$. Let $\tilde{A}=\left(\mu_{\tilde{A}}(x), v_{\tilde{A}}(x)\right)$ be an IFN, a score function $S$ of an intuitionistic fuzzy number is represented as follows.

$$
S(\tilde{A})=\mu_{\tilde{A}}(x)-v_{\tilde{A}}(x), S(\tilde{A}) \in[-1,1]
$$

Definition 2.5. [55] The accuracy function $H$ defined as sum of the membership function $\left(\mu_{\tilde{A}}(x)\right)$ and the non-membership function $\left(v_{\tilde{A}}(x)\right)$. Let $\tilde{A}=\left(\mu_{\tilde{A}}(x), v_{\tilde{A}}(x)\right)$ be an IFN, an accuracy function $H$ of an intuitionistic fuzzy number is represented as follows.

$$
H(\tilde{A})=\mu_{\tilde{A}}(x)+v_{\tilde{A}}(x), H(\tilde{A}) \in[0,1]
$$

Definition 2.6. [56,57] A simple method was introduced to compare any two IFNs $\tilde{A}=\left(\mu_{\tilde{A}}(x), v_{\tilde{A}}(x)\right)$ and $\tilde{B}=\left(\mu_{\tilde{B}}(x), v_{\tilde{B}}(x)\right)$ as below:

If $S(\tilde{A})<S(\tilde{B})$, then $\tilde{A}<\tilde{B}$;

If $S(\tilde{A})=S(\tilde{B})$, and

If $H(\tilde{A})=H(\tilde{B})$, then $\tilde{A}=\tilde{B}$;

If $H(\tilde{A})<H(\tilde{B})$, then $\tilde{A}<\tilde{B}$.

Definition 2.7. [56,57] Some basic aggregation operators for IFNs $\left(\tilde{A}_{1}, \tilde{A}_{2}, \ldots, \tilde{A}_{n}\right)$ were developed by using the weight vector $w=\left(w_{1}, w_{2}, \ldots, w_{n}\right)^{T}$ of IFNs under condition $w_{i} \in[0,1]$ and $\sum_{i=1}^{n} w_{i}=1$.

(a) Intuitionistic fuzzy weighted averaging (IFWA) operator

$$
I F W A_{w}\left(\tilde{A}_{1}, \tilde{A}_{2}, \ldots, \tilde{A}_{n}\right)=\sum_{i=1}^{n} \oplus_{w_{i}} \tilde{A}_{i}=\left(1-\prod_{i=1}^{n}\left(1-\mu_{i}\right)^{w_{i}}, \prod_{i=1}^{n} v_{i}^{w_{i}}\right)
$$

(b) Intuitionistic fuzzy weighted geometric (IFWG) operator

$$
I F W G_{w}\left(\tilde{A}_{1}, \tilde{A}_{2}, \ldots ., \tilde{A}_{n}\right)=\sum_{i=1}^{n} \otimes \tilde{A}_{i}^{w_{i}}=\left(\prod_{i=1}^{n} \mu_{i}^{w_{i}}, 1-\prod_{i=1}^{n}\left(1-v_{i}\right)^{w_{i}}\right)
$$

Definition 2.8. [58,59] The entropy is a measure of the fuzziness Fuzzy entropy which derives from the concept of probability and measures the discrimination of attributes has been introduced by Zadeh. However, the intuitionistic fuzzy entropy becomes distinct from traditional entropy due to the fact that it explains the data's credibility. Researchers have developed several entropy measures for intuitionistic fuzzy sets. Under the assumption that $\tilde{A}$ be an intuitionistic fuzzy set in the universe of discourse $X=\left\{x_{1}, x_{2}, . ., x_{n}\right\}$ some of the entropy measures are presented below.

(1) Burillo \& Bustince [60]

$$
E_{B B}=\sum_{i=1}^{n}\left(1-\left(\mu_{i}+v_{i}\right)\right)=\sum_{i=1}^{n} \pi_{i}
$$

(2) Szmidt \& Kacprzyk, [5]

$E_{S K}=\frac{1}{n} \sum_{i=1}^{n} \frac{1-\left(\mu_{i} \cap \pi_{i}\right)}{1-\left(\mu_{i} \cup \pi_{i}\right)}$

(3) De Luca \& Termini [61]

$E_{L T}=-\frac{1}{n \ln 2} \sum_{i}^{n_{i}}\left[\mu_{i} \ln \left(\frac{\mu_{i}}{\mu_{i}+v_{i}}\right)+v_{i} \ln \left(\frac{v_{i}}{\mu_{i}+v_{i}}\right)-\pi_{i} \ln 2\right]$

(4) Vlachos \& Sergiadis [15]

$$
E_{V S}=\sum_{i}^{n_{i}}\left[\begin{array}{l}
\mu_{\tilde{A}}\left(x_{i}\right) \ln \left(\frac{\mu_{\tilde{A}}\left(x_{i}\right)}{(1 / 2)\left(\mu_{\tilde{A}}\left(x_{i}\right)+\mu_{\tilde{B}}\left(x_{i}\right)\right)}\right)+ \\
v_{\tilde{A}}\left(x_{i}\right) \ln \left(\frac{\mu_{\tilde{A}}\left(x_{i}\right)}{(1 / 2)\left(v_{\tilde{A}}\left(x_{i}\right)+v_{\tilde{B}}\left(x_{i}\right)\right)}\right)
\end{array}\right]
$$

\section{Multi-Criteria Group Evaluation Framework}

In this section, the proposed approach to multi-person and multiattribute intuitionistic fuzzy evaluation is introduced. This approach consists of eight steps divided into the three main processes (preparation, integration and evaluation) as shown in Table 1 . In addition, abbreviations and symbols utilized for the approach are presented in Table 2 .

\footnotetext{
Table 1. The framework of the proposed approach

Preparation

Step 1. Determine the factors, sub-factors, and attributes

Step 2. Determine the characteristic of each expert group

Step 3. Calculate the weights of each expert group

Step 4. Aggregate the evaluators' opinions using the intuitionistic fuzzy
} 
weighted geometric (IFWG) operator

Step 5. Calculate the weights of each factor and sub-factor using the entropy

Integration

Step 6. Integrate factors and sub-factors weights into the score function Evaluation

Step 7. Evaluate the alternative with linguistic variables

Step 8. Calculate the score value

Table 2. Abbreviations and symbols.

Abbreviations

\begin{tabular}{ll} 
AF & Accuracy function \\
GTS & General technical skills \\
Att & Attribute \\
SF & Score function \\
NTS & Non-technical skills \\
\hline Symbols & \\
\hline$A_{a}$ & accuracy for $\mathrm{a}^{\text {th }}$ alternative \\
$A t t_{k}^{i(j)}$ & the $\mathrm{k}^{\text {th }}$ attribute of the $\mathrm{j}^{\text {th }}$ sub-factor of the $\mathrm{i}^{\text {th }}$ factor \\
$F_{i}$ & the ith factor \\
$F_{i}^{g(l)}$ & the IFN assigned for the opinion of the $\mathrm{l}^{\text {th }}$ evaluator in the \\
$n_{k}^{i(j)}$ & $\begin{array}{l}\text { th } \\
\text { toxpert group for the } \mathrm{i}^{\text {th }} \text { factor }\end{array}$ \\
$n_{l}^{g}$ & total number of attributes in $\mathrm{j}^{\text {th }}$ sub-factors of the $\mathrm{i}^{\text {th }}$ factor \\
$S_{a}$ & score for $\mathrm{a}^{\text {th }}$ alternative \\
$S B F_{i(j)}$ & the $\mathrm{j}^{\text {th }}$ sub-factor of the $\mathrm{i}^{\text {th }}$ factor \\
$n_{i(j)}$ & total number of sub-factors in the $\mathrm{i}^{\text {th }}$ factor \\
$w_{g}$ & the weight of the $\mathrm{g}^{\text {th }}$ expert group \\
$\lambda_{i}$ & the weight of the $\mathrm{i}^{\text {th }}$ factor \\
$\beta_{i(j)}$ & the weight of the $\mathrm{j}^{\text {th }}$ sub-factor of the $\mathrm{i}^{\text {th }}$ factor \\
\hline
\end{tabular}

Step 1: Determine the factors, sub-factors, attributes and design the evaluation scale of IFS.

While determining factors and sub-factors, two rules should be paid attention: (i) every factor should have a sub-factor (ii) every sub-factor should have an attribute. In addition, the expressions used while specifying factors, sub-factors, and attributes should be inclusive, simple, and without repetition.

Step 2: Determine the characteristic of each expert group.

The knowledge and experience of experts are different. For this reason, the ratings of importance are determined for experts by taking certain criteria into consideration. If the number of experts is low, the rating of importance can be determined for each expert. But in cases where the number of experts is high, the determination process of the rating of importance can be complex. If a group evaluation is carried out with a large number of experts, the experts should be grouped according to certain criteria. In this step, it needs to note that a different number of evaluators can exist in each expert group.

Step 3: Calculate the weights of each expert group.

Boran et al. [9] showed that the rating of importance of experts stated as IFNs can be normalized and converted to weight with the following equation. Let $\tilde{G}_{g}=\left(\mu_{g}, v_{g}, \pi_{g}\right)$ be an IFN for rating of $\mathrm{g}^{\text {th }}$ expert group. The weight of $\mathrm{g}^{\text {th }}$ expert group can be obtained as:

$$
w_{g}=\frac{\mu_{g}+\pi_{g}\left(\frac{\mu_{g}}{\mu_{g}+v_{g}}\right)}{\sum_{g=1}^{n}\left(\mu_{g}+\pi_{g}\left(\frac{\mu_{g}}{\mu_{g}+v_{g}}\right)\right)}, w_{g} \geq 0 \text { and } \sum_{g=1}^{n} w_{g}=1
$$

Step 4: Aggregate the evaluators' opinions using the intuitionistic fuzzy weighted geometric (IFWG) operator.

In the evaluation process with different groups, firstly all the individual evaluators' opinions are aggregated as a group opinion within their own group. Then, all group opinions are fused into as a group opinion to calculate weights of factor and subfactor. In order to do that, the IFWG operator proposed by $\mathrm{Xu}$ [57] is adapted to the multi-group evaluation. Opinions in the same group are aggregated under the assumption that their weights $\left(1 / n_{l}^{g}\right)$ are equal.

$$
I F W G=\left(\mu_{i}^{g}, v_{i}^{g}\right)=\left(\left(\prod_{l=1}^{n_{l}^{g}}\left(\mu_{i}^{g_{g}(l)}\right) \frac{1}{n_{l}^{g}}\right)^{w_{g}}, 1-\left(\prod_{l=1}^{n_{l}^{g}}\left(1-v_{i}^{g}(l)\right) \frac{1}{n_{l}^{g}}\right)^{w_{g}}\right)
$$

Step 5: Calculate the weights of each factor and sub-factor using the entropy.

In this step following intuitionistic fuzzy entropy measure given by De Luca and Termini [61] is adapted to obtain the weight vector of factors $\lambda_{i}=\left(\lambda_{1}, \lambda_{2}, \ldots, \lambda_{n}\right)$ and weight vector of sub-factors $\beta_{i(j)}=\left(\beta_{1(1)}, \beta_{1(2)}, \ldots, \beta_{2(1)}, \beta_{2(2)}, \ldots, \beta_{n_{i}\left(n_{j}\right)}\right) \quad$ where $\quad \lambda_{i}, \beta_{i(j)} \in[0,1]$ and $\sum_{i=1}^{n_{i}} \lambda_{K_{i}}=1, \sum_{j=1}^{n_{j}} \beta_{i(j)}=1$. Let $\tilde{F}_{i}^{g}=\left(\mu_{i}^{g}, v_{i}^{g}, \pi_{i}^{g}\right)$ be an IFN of the aggregated rating of importance of the $\mathrm{g}^{\text {th }}$ expert group for the $\mathrm{i}^{\text {th }}$ factor. Adapted entropy measures for factor and sub-factor are presented respectively as below.

$$
\begin{aligned}
& E_{i}=-\frac{1}{n \ln 2} \sum_{g=1}^{n_{g}}\left[\mu_{i}^{g} \ln \left(\frac{\mu_{i}^{g}}{\mu_{i}^{g}+v_{i}^{g}}\right)+v_{i} \ln \left(\frac{v_{i}^{g}}{\mu_{i}^{g}+v_{i}^{g}}\right)-\pi_{i}^{g} \ln 2\right] \\
& E_{i(j)}=-\frac{1}{n \ln 2} \sum_{g=1}^{n_{g}}\left[\mu_{i(j)}^{g} \ln \left(\frac{\mu_{i(j)}^{g}}{\mu_{i(j)}^{g}+v_{i(j)}^{g}}\right)+v_{i} \ln \left(\frac{v_{i(j)}^{g}}{\mu_{i(j)}^{g}+v_{i(j)}^{g}}\right)-\pi_{i(j)}^{g} \ln 2\right]
\end{aligned}
$$

After the entropy values are calculated, the weights of factors and sub-factors are calculated with the equation given below [62].

$$
\begin{aligned}
& \lambda_{i}=\frac{1-E_{i}}{n_{i}-\sum_{i=1}^{n_{i}} E_{i}} \\
& \beta_{i(j)}=\frac{1-E_{i(j)}}{n_{i(j)}-\sum_{j=1}^{n_{i(j)}} E_{i(j)}}
\end{aligned}
$$

Step 6: Integrate factors and subfactors weights into the score function.

The evaluation score is formed by integrating factor weight, subfactor weight, number of factors, number of sub-factors and number of attributes into score function. Let $\tilde{D}_{k}^{i(j)}=\left(\mu_{k}^{i(j)}, v_{k}^{i(j)}\right)$ be the intuitionistic fuzzy number represented to importance rating of the opinion stated for the $\mathrm{k}^{\text {th }}$ attribute of the $\mathrm{j}^{\text {th }}$ sub-factor of the $\mathrm{i}^{\text {th }}$ factor. The score function $(S F()$.$) , and/or the accuracy$ function $(A F()$.$) which is used when the score values are equal$ are presented below. 


$$
\begin{aligned}
& S F=\left(\begin{array}{l}
\prod_{i=1}^{n_{i}}\left(\prod_{j=1}^{n_{i(j)}}\left(\prod_{k=1}^{n_{k}^{i(j)}}\left(\mu_{k}^{i(j)}\right)^{\frac{1}{n_{k}^{i(j)}}}\right)^{\beta_{i(j)}}\right)^{\lambda_{i}}- \\
\left(1-\left(\prod_{i=1}^{n_{i}}\left(\prod_{j=1}^{n_{i(j)}}\left(\prod_{k=1}^{n_{k}^{i(j)}}\left(1-v_{k}^{i(j)}\right)^{\frac{1}{n_{k}^{i(j)}}}\right)^{\beta_{i(j)}}\right)\right)^{\lambda_{i}}\right)
\end{array}\right) \\
& A F=\left(\begin{array}{l}
\prod_{i=1}^{n_{i}}\left(\prod_{j=1}^{n_{i(j)}}\left(\prod_{k=1}^{n_{k}^{i(j)}}\left(\mu_{k}^{i(j)}\right)^{\frac{1}{n_{k}^{i(j)}}}\right)^{\beta_{i(j)}}\right)^{\lambda_{i}}+ \\
\left(1-\left(\prod_{i=1}^{n_{i}}\left(\prod_{j=1}^{n_{i(j)}}\left(\prod_{k=1}^{n_{k}^{i_{k}(j)}}\left(1-v_{k}^{i(j)}\right)^{\frac{1}{n_{k}^{(i j)}}}\right)^{\beta_{i(j)}}\right)\right)^{\lambda_{i}}\right)
\end{array}\right)
\end{aligned}
$$

In order to evaluate alternatives on a factor basis, the score function $\left(S F_{i}().\right)$, and/or accuracy function $\left(A F_{i}().\right)$ are presented below is used to aggregate the opinions stated for the attributes on a factor basis.

$$
\begin{aligned}
& S F_{i}=\left(\begin{array}{l}
\prod_{j=1}^{n_{i(j)}}\left(\prod_{k=1}^{n_{k}^{i(j)}}\left(\mu_{k}^{i(j)}\right)^{\frac{1}{n_{k}^{i(j)}}}\right)^{\beta_{i(j)}}- \\
\left(1-\left(\prod_{j=1}^{n_{i(j)}}\left(\prod_{k=1}^{n_{k}^{i(j)}}\left(1-v_{k}^{i(j)}\right)^{\frac{1}{i_{k}^{(i j)}}}\right)^{\beta_{i(j)}}\right)\right)
\end{array}\right) \\
& A F_{i}=\left(\begin{array}{l}
\prod_{j=1}^{n_{i(j)}}\left(\prod_{k=1}^{n_{k}^{i(j)}}\left(\mu_{k}^{i(j)}\right)^{\frac{1}{i_{k}^{(i j)}}}\right)^{\beta_{i(j)}}+ \\
\left(1-\left(\prod_{j=1}^{n_{i(j)}}\left(\prod_{k=1}^{n_{k}^{i(j)}}\left(1-v_{k}^{i(j)}\right)^{\frac{1}{n_{k}^{i(j)}}}\right)^{\beta_{i(j)}}\right)\right)
\end{array}\right)
\end{aligned}
$$

If the alternatives are evaluated with more than one evaluator's opinion, score function, and/or accuracy function presented below is used. Let $\tilde{D}_{l}^{i, j(k)}=\left(\mu_{l}^{i, j(k)}, v_{l}^{i, j(k)}\right)$ be the intuitionistic fuzzy number represented to importance rating of the opinion stated by the $1^{\text {th }}$ evaluator for the $\mathrm{k}^{\text {th }}$ attribute of the $\mathrm{j}^{\text {th }}$ sub-factor of the $\mathrm{i}^{\text {th }}$ factor. Score and accuracy functions used for group evaluation are presented as follows.

$$
\begin{aligned}
& S F=\left(\begin{array}{l}
\prod_{l=1}^{n_{l}}\left(\prod_{i=1}^{n_{i}}\left(\prod_{j=1}^{n_{i(j)}}\left(\prod_{k=1}^{n_{k}^{i(j)}}\left(\mu_{l}^{i, j(k)}\right)^{\frac{1}{n_{k}^{i(j)}}}\right)^{\beta_{i(j)}}\right)^{\lambda_{i}}\right)^{w_{l}}- \\
\left(1-\prod_{l=1}^{n_{l}}\left(\prod_{i=1}^{n_{i}}\left(\prod_{j=1}^{n_{i(j)}}\left(\prod_{k=1}^{n_{k}^{i(j)}}\left(1-v_{l}^{i, j(k)}\right)^{\frac{1}{i_{k}^{(i j)}}}\right)^{\beta_{i(j)}}\right)\right)^{\lambda_{i}}\right)^{w_{l}}
\end{array}\right) \\
& A F=\left(\begin{array}{l}
\prod_{l=1}^{n_{l}}\left(\prod_{i=1}^{n_{i}}\left(\prod_{j=1}^{n_{i(j)}}\left(\prod_{k=1}^{n_{k}^{(i j)}}\left(\mu_{l}^{i, j(k)}\right)^{\frac{1}{n_{k}^{(j)}}}\right)^{\beta_{i(j)}}\right)^{\lambda_{i}}\right)^{w_{l}}+ \\
\left(1-\prod_{l=1}^{n_{l}}\left(\prod_{i=1}^{n_{i}}\left(\prod_{j=1}^{n_{i(j)}}\left(\prod_{k=1}^{n_{k}^{(i j)}}\left(1-v_{l}^{i, j(k)}\right)^{\frac{1}{n_{k}^{i(j)}}}\right)^{\beta_{i(j)}}\right)\right)^{\lambda_{i}}\right)^{w_{l}}
\end{array}\right)
\end{aligned}
$$

Step 7: Evaluate the alternatives with linguistic variables.

The evaluation of alternatives is done individually or by the group. If alternatives are evaluated by more than one evaluator, weights should be determined for these evaluators by using the scale of IFS formed in Step 2.

Step 8: Calculate the score value.

In this step, the score value is calculated. If there are more than one alternative, it is made a comparison with the following rules using score and accuracy values. Let $S_{1}$ and $S_{2}$ be two intuitionistic fuzzy group evaluation score values of alternative 1 and alternative 2 .

If $S_{1}>S_{2}$, then Alternative 1 is better than Alternative 2;

If $S_{1}<S_{2}$, then Alternative 2 is better than Alternative 1;

If $S_{1}=S_{2}$ and If $A_{1}=A_{2}$, then Alternative 1 and Alternative 2 are equal;

If $S_{1}=S_{2}$ and If $A_{1}>A_{2}$, then Alternative 1 is better than Alternative 2;

If $S_{1}=S_{2}$ and If $A_{1}<A_{2}$, then Alternative 2 is better than Alternative 1 .

\section{Numerical Example}

After having presented the proposed evaluation model, in this section, an illustrative example is given for the application of the proposed method to assess the level of T-shaped skills of engineers and to select the best possible engineer among the candidates for the company. Headquartered in Germany and had more than 500 employees, this retail company provides services with more than twenty thousand varieties for home products to European countries such as France, Netherlands, England, Austria, Luxemburg, Denmark, Belgium, Italy, Spain, Sweden, Finland.

For the process analyst position, this company wants to employ an engineer with $\mathrm{T}$-shaped engineer skills that many big companies such as IBM, IDEO, P\&G, Nike expect from engineers. Five of the candidates who apply for the job advertisement are invited to the interview. The firm conducts a detailed interview for the features it expects for the position of process analyst. In addition, the firm desires to assess the Tshaped engineering skills for candidates.

Step 1: Determine the factors, sub-factors, and attributes.

Based on job-advertisements analysis for engineers and an extensive literature review of general technical and non-technical (soft) skills that T-shaped engineers should acquire, 39 evaluation attributes are determined (Appendix). 7 of these attributes are general technical skills and 32 of them are non-technical skills. Then non-technical (soft) attributes are grouped into five subfactors based on the review [63-66]. Factors and sub-factors are given in Table 3 .

Table 3. Factors and sub-factors

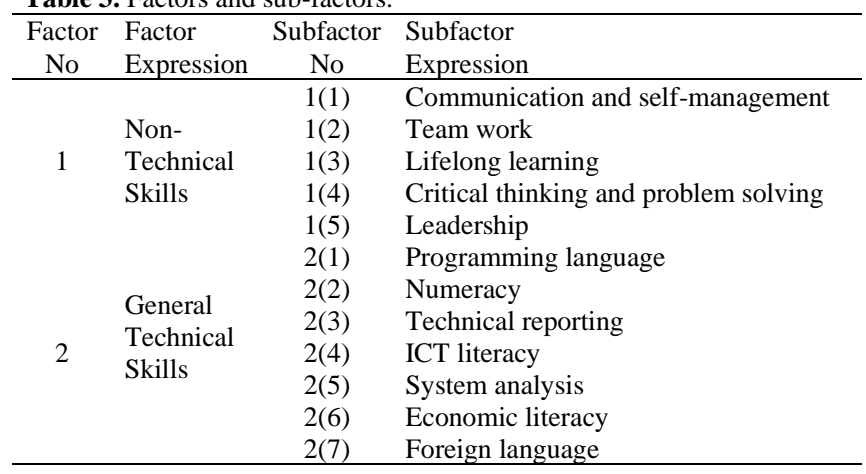


Step 2: Determine the characteristic of each expert group.

After determining factors, sub-factors and attributes, twenty-five experts are assessed considering several criteria including job field, job tenure, education, company type, organization size. Thirteen of the experts have a master's degree in several engineering programs and three of them have been working as a manager in large-sized companies, two of them have been working as an engineer in large-sized companies, four of them have been working as a manager in government agencies, four of them have been working as a manager in small and medium-sized companies for more than 5 years. Five of the experts have a doctoral degree in several engineering programs and two of them have been working as a manager in large-sized companies, one of them has been working as a manager in government agencies, two of them have been working as an engineer in large-sized companies for more than 5 years. Linguistic terms used for the ratings of the experts' groups, group characteristics, and the number of experts for each group are given in Table 4.

Step 3: Calculate the weights of each expert group.

With the aim to determine the weights of the experts' groups the model introduced by Boran et al. [9] is used. The weights of experts' groups are obtained as $0.206,0.195,0.183,0.163,0.138$, 0.115 . The weights of expert 1 and expert 2, for example, are computed as

$$
\begin{aligned}
& w_{1}=\frac{1.00+0.00\left(\frac{1.00}{1.00+0.00}\right)}{\left(1.00+0.00\left(\frac{1.00}{1.00+0.00}\right)\right)+\ldots . .+\left(0.50+0.10\left(\frac{0.50}{0.50+0.40}\right)\right)}=0.206 \\
& w_{2}=\frac{0.90+0.05\left(\frac{0.90}{0.90+0.05}\right)}{\left(1.00+0.00\left(\frac{1.00}{1.00+0.00}\right)\right)+\ldots . .+\left(0.50+0.10\left(\frac{0.50}{0.50+0.40}\right)\right)}=0.195
\end{aligned}
$$

Step 4: Aggregate the evaluators' opinions using the intuitionistic fuzzy weighted geometric (IFWG) operator.

The experts determine the importance rating of factors and subfactors using linguistic terms presented in Table 5. The ratings assigned by the experts to factors and subfactors are shown in respectively Table 6 and Table 7.

Table 5. Linguistic terms for rating the importance of factors, sub-factors and attributes (Zhang \& Liu, 2011).

\begin{tabular}{lc}
\hline Definition of linguistic terms & IFNs \\
\hline Extreme Low (EL) & $(0.05,0.95)$ \\
Very Low (VL) & $(0.15,0.80)$ \\
Low (L) & $(0.25,0.65)$ \\
Medium Low (ML) & $(0.35,0.55)$ \\
Medium (M) & $(0.50,0.40)$ \\
Medium High (MH) & $(0.65,0.25)$ \\
High (H) & $(0.75,0.15)$ \\
Very High (VH) & $(0.85,0.10)$ \\
Extreme High (EH) & $(0.95,0.05)$ \\
\hline
\end{tabular}

Table 6. The ratings of the factors.

\begin{tabular}{cccccccc}
\hline$g=\#$ & $l(\#)$ & $F_{1}$ & $F_{2}$ & $g=\#$ & $l(\#)$ & $F_{1}$ & $F_{2}$ \\
\hline \multirow{3}{*}{$g=1$} & 1 & EH & MH & & 1 & L & VL \\
& 2 & M & VH & $g=4$ & 2 & VL & VH \\
& 3 & VH & M & & 3 & M & VH \\
\hline
\end{tabular}

\begin{tabular}{cccccccc}
\hline \multirow{6}{*}{$g=2$} & 4 & M & M & & 4 & H & M \\
& 5 & H & ML & & 5 & L & M \\
& 1 & H & VL & & 1 & VH & VL \\
& 2 & VL & VL & \multirow{6}{*}{$g=5$} & 2 & EH & VH \\
& 3 & M & M & & 3 & H & VH \\
& 4 & H & L & & 4 & VL & M \\
& 1 & L & M & & 1 & H & M \\
& 2 & L & L & $g=6$ & 2 & H & H \\
& 3 & VL & VH & & 3 & VL & M \\
& 4 & M & VH & & & & \\
\hline
\end{tabular}

After transferring linguistic terms into the corresponding IFNs with respect to Table 5, the experts' personal assessments are aggregated into a collective form for each group by using IFWG operator. Aggregated assessments of each group for factors are shown in Table 8, for sub-factors are shown in Table 9 and Table 10 .

Table 7. The ratings of the sub-factors.

\begin{tabular}{ccccccc}
\hline & $l(\#)$ & $S B F_{1(1)}$ & $S B F_{1(2)}$ & $S B F_{1(3)}$ & $S B F_{1(4)}$ & $S B F_{1(5)}$ \\
\hline \multirow{5}{*}{$g=1$} & 1 & EH & EH & EH & VH & VH \\
& 2 & MH & MH & MH & H & VH \\
& 3 & M & MH & EH & MH & VH \\
& 4 & VH & M & MH & MH & M \\
& 5 & VH & EH & EH & EH & VH \\
\hline \multirow{5}{*}{$g=2$} & 1 & H & EH & EH & H & H \\
& 2 & VH & MH & MH & MH & H \\
& 3 & EH & M & MH & MH & M \\
& 4 & M & M & MH & MH & M \\
& 5 & EH & H & M & H & MH \\
\hline \multirow{5}{*}{$g=3$} & 1 & M & MH & H & MH & H \\
& 2 & M & EH & EH & EH & EL \\
& 3 & M & MH & MH & EH & M \\
& 4 & M & MH & L & H & VL \\
\hline \multirow{5}{*}{$g=4$} & 1 & H & M & MH & H & H \\
& 2 & H & EH & L & H & VL \\
& 3 & VL & VH & MH & MH & M \\
& 4 & EH & M & EH & EH & EH \\
\hline \multirow{5}{*}{$g=5$} & 1 & EH & H & M & H & L \\
& 2 & VH & MH & MH & H & H \\
& 3 & VH & VL & L & M & M \\
& 4 & MH & EL & EH & EH & VH \\
\hline \multirow{2}{*}{$g=6$} & 1 & H & MH & EH & H & L \\
& 2 & MH & M & L & M & M \\
& 3 & H & M & MH & EH & VL \\
\hline \multirow{6}{*}{} & & & & & & \\
& & & & & & \\
& & & & &
\end{tabular}

Table 8. Aggregated assessments of each of the factors.

\begin{tabular}{lcc}
\hline & $F_{1}$ & $F_{2}$ \\
\hline$g=1$ & $(0.925,0.054)$ & $(0.883,0.087)$ \\
$g=2$ & $(0.947,0.040)$ & $(0.973,0.020)$ \\
$g=3$ & $(0.865,0.106)$ & $(0.764,0.196)$ \\
$g=4$ & $(0.907,0.076)$ & $(0.888,0.091)$ \\
$g=5$ & $(0.831,0.135)$ & $(0.920,0.059)$ \\
$g=6$ & $(0.910,0.071)$ & $(0.938,0.044)$ \\
\hline
\end{tabular}

Step 5: Calculate the weights of each factor and sub-factor using the entropy.

The weight of factors and sub-factors is determined based on the entropy model after aggregated assessments of each group. The

\begin{tabular}{|c|c|c|c|c|}
\hline $\begin{array}{l}\text { Group } \\
\text { No }\end{array}$ & Group Specifications & Linguistic terms & IFNs & $\begin{array}{c}\text { Number of } \\
\text { experts }\end{array}$ \\
\hline 1 & manager in a large sized company; $\mathrm{MSc}$ or $\mathrm{PhD}$ degree; 5+ years of experience & Extreme High $(\mathrm{EH})$ & $(1.00,0.00)$ & 5 \\
\hline 2 & manager in a public company; MSc or PhD degree; $5+$ years of experience & Very High (VH) & $(0.90,0.0)$ & 5 \\
\hline 3 & $\begin{array}{l}\text { manager in a small and medium sized company; MSc or PhD degree; } 5+\text { years } \\
\text { of experience }\end{array}$ & High $(\mathrm{H})$ & $(0.80,0.10)$ & 4 \\
\hline 4 & engineer in a large-sized company; MSc or $\mathrm{PhD}$ degree; 5+ years of experience & Medium High (MH) & $(0.75,0.20)$ & 4 \\
\hline 5 & $\begin{array}{l}\text { manager in a small and medium sized company; bachelor degree; } 3+\text { years of } \\
\text { experience }\end{array}$ & Medium (M) & $(0.60,0.30)$ & 4 \\
\hline 6 & engineer in all company; bachelor degree; $3+$ years of experience & Medium Low (ML) & $(0.50,0.40)$ & 3 \\
\hline
\end{tabular}

Table 4. Experts' groups profile and related IFNs. 
entropy values of factors and sub-factors are calculated by utilizing De Luca \& Termini [61]'s entropy measure for factor and sub-factor. After the entropy values are obtained, the weights of factors and sub-factors are calculated and presented shown in Table 11, Table 12 and Table 13. The weights of factors are obtained as $\lambda_{1}=0.532$ and $\lambda_{2}=0.468$ for non-technical (soft) skills and general technical skills, respectively. The weights of the subfactors of the non-technical skill factor are calculated as $\beta_{1(j)}=(0.223,0.185,0.197,0.250,0.144)$ and the weights of the subfactors of the general technical skill factor are calculated as $\beta_{2(j)}=(0.137,0.149,0.153,0.130,0.143,0.138,0.150)$.

Table 11. Entropy value and weight of each of the factors

\begin{tabular}{ccc}
\hline & $F_{1}$ & $F_{2}$ \\
\hline$g=1$ & -0.223 & -0.314 \\
$g=2$ & -0.176 & -0.102 \\
$g=3$ & -0.355 & -0.514 \\
$g=4$ & -0.279 & -0.317 \\
$g=5$ & -0.415 & -0.238 \\
$g=6$ & -0.269 & -0.193 \\
\hline$n_{i}\left(E_{i}^{g}\right)$ & -1.716 & -1.677 \\
\hline$E_{i}$ & 1.238 & 1.210 \\
\hline$\lambda_{i}$ & 0.532 & 0.468 \\
\hline
\end{tabular}

Table 12. Entropy value and weight of each of the sub-factors affiliated $1^{\text {nd }}$ factor.

\begin{tabular}{cccccc}
\hline & $S B F_{1(1)}$ & $S B F_{1(2)}$ & $S B F_{1(3)}$ & $S B F_{1(4)}$ & $S B F_{1(5)}$ \\
\hline$g=1$ & -0.188 & -0.204 & -0.141 & -0.168 & -0.171 \\
$g=2$ & -0.162 & -0.242 & -0.231 & -0.206 & -0.258 \\
$g=3$ & -0.336 & -0.195 & -0.281 & -0.134 & -0.541 \\
$g=4$ & -0.302 & -0.227 & -0.289 & -0.155 & -0.337 \\
$g=5$ & -0.129 & -0.508 & -0.305 & -0.182 & -0.298 \\
$g=6$ & -0.171 & -0.284 & -0.292 & -0.179 & -0.455 \\
\hline$n_{g}\left(E_{1(j)}^{g}\right)$ & -1.287 & -1.659 & -1.539 & -1.024 & -2.061 \\
$\sum_{g=1}$ & & & & & \\
\hline$E_{1(j)}$ & 0.371 & 0.479 & 0.444 & 0.295 & 0.595 \\
\hline$\beta_{1(j)}$ & 0.223 & 0.185 & 0.197 & 0.250 & 0.144 \\
\hline
\end{tabular}

Step 6: Integrate factors and subfactors weights into the score function.

The data obtained in the preparation phase, which includes the first five steps, is integrated into the score function and an evaluation model is formed. The score function used to ranking and selecting of the alternatives is based on relative comparisons where the first parameter (aggregated degree of membership) is the higher, the second parameter (aggregated degree of nonmembership) is the lower. The score function for this application is obtained as below.

$S F=$

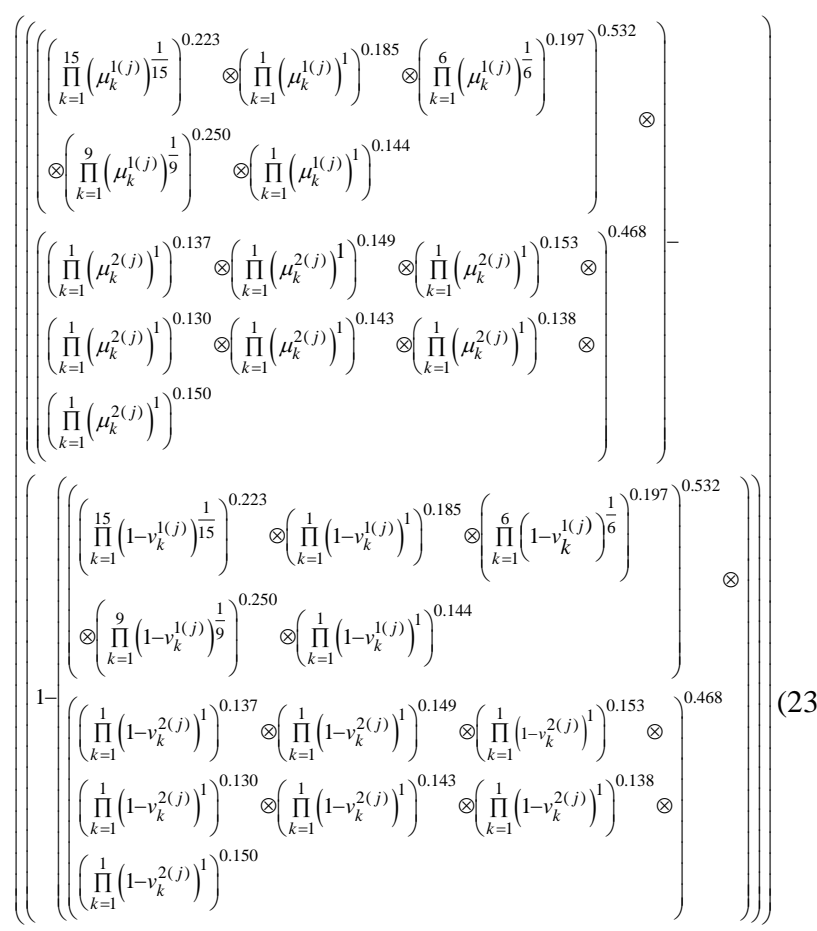

Step 7: Evaluate the alternative with linguistic variables.

The assessments are carried out by the human resources specialist considering the 39 attributes (skills) determined in this study for the five engineers.

Step 8: Calculate the score and accuracy value.

In order to evaluate and rank alternatives, score values related to alternatives are calculated. In case the score value is equal, a

Table 9. Aggregated assessments of sub-factors affiliated $1^{\text {th }}$ factor.

\begin{tabular}{cccccc}
\hline & $S B F_{1(1)}$ & $S B F_{1(2)}$ & $S B F_{1(3)}$ & $S B F_{1(4)}$ & $S B F_{1(5)}$ \\
\hline$g=1$ & $(0.940,0.043)$ & $(0.934,0.048)$ & $(0.959,0.030)$ & $(0.945,0.036)$ & $(0.946,0.038)$ \\
$g=2$ & $(0.953,0.036)$ & $(0.919,0.061)$ & $(0.924,0.057)$ & $(0.930,0.048)$ & $(0.908,0.065)$ \\
$g=3$ & $(0.881,0.100)$ & $(0.940,0.046)$ & $(0.906,0.077)$ & $(0.963,0.028)$ & $(0.764,0.229)$ \\
$g=4$ & $(0.902,0.087)$ & $(0.937,0.059)$ & $(0.911,0.083)$ & $(0.955,0.034)$ & $(0.887,0.103)$ \\
$g=5$ & $(0.970,0.028)$ & $(0.822,0.220)$ & $(0.915,0.093)$ & $(0.955,0.045)$ & $(0.916,0.090)$ \\
$g=6$ & $(0.959,0.041)$ & $(0.930,0.086)$ & $(0.931,0.091)$ & $(0.961,0.045)$ & $(0.859,0.183)$ \\
\hline
\end{tabular}

Table 10. Aggregated assessments of sub-factors affiliated $2^{\text {nd }}$ factor.

\begin{tabular}{cccccccc}
\hline & $S B F_{2(1)}$ & $S B F_{2(2)}$ & $S B F_{2(3)}$ & $S B F_{2(4)}$ & $S B F_{2(5)}$ & $S B F_{2(6)}$ & $S B F_{2(7)}$ \\
\hline$g=1$ & $(0.940,0.043)$ & $(0.951,0.031)$ & $(0.980,0.015)$ & $(0.864,0.102)$ & $(0.947,0.033)$ & $(0.905,0.066)$ & $(0.989,0.036)$ \\
$g=2$ & $(0.920,0.056)$ & $(0.958,0.027)$ & $(0.972,0.018)$ & $(0.847,0.116)$ & $(0.934,0.043)$ & $(0.906,0.066)$ & $(0.981,0.014)$ \\
$g=3$ & $(0.893,0.077)$ & $(0.965,0.022)$ & $(0.981,0.014)$ & $(0.892,0.080)$ & $(0.930,0.046)$ & $(0.902,0.070)$ & $(0.953,0.030)$ \\
$g=4$ & $(0.932,0.047)$ & $(0.968,0.022)$ & $(0.973,0.017)$ & $(0.856,0.110)$ & $(0.948,0.034)$ & $(0.918,0.058)$ & $(0.978,0.015)$ \\
$g=5$ & $(0.904,0.072)$ & $(0.973,0.016)$ & $(0.985,0.011)$ & $(0.906,0.070)$ & $(0.934,0.048)$ & $(0.934,0.046)$ & $(0.973,0.016)$ \\
$g=6$ & $(0.887,0.086)$ & $(0.967,0.021)$ & $(0.994,0.006)$ & $(0.911,0.067)$ & $(0.952,0.034)$ & $(0.947,0.036)$ & $(0.967,0.019)$ \\
\hline
\end{tabular}


Table 13. Entropy value and weight of each of the sub-factors affiliated $2^{\text {nd }}$ factor.

\begin{tabular}{cccccccc}
\hline & $S B F_{2(1)}$ & $S B F_{2(2)}$ & $S B F_{2(3)}$ & $S B F_{2(4)}$ & $S B F_{2(5)}$ & $S B F_{2(6)}$ & $S B F_{2(7)}$ \\
\hline$g=1$ & -0.188 & -0.151 & -0.081 & -0.350 & -0.159 & -0.261 & -0.139 \\
$g=2$ & -0.231 & -0.135 & -0.098 & -0.380 & -0.192 & -0.261 & -0.077 \\
$g=3$ & -0.290 & -0.114 & -0.078 & -0.296 & -0.201 & -0.272 & -0.146 \\
$g=4$ & -0.204 & -0.113 & -0.093 & -0.367 & -0.160 & -0.237 & -0.082 \\
$g=5$ & -0.274 & -0.091 & -0.062 & -0.269 & -0.203 & -0.200 & -0.091 \\
$g=6$ & -0.310 & -0.110 & -0.036 & -0.260 & -0.158 & -0.166 & -0.102 \\
\hline$\sum_{g=1}^{g}\left(E_{2(j)}^{g}\right)$ & -1.498 & -0.714 & -0.448 & -1.922 & -1.074 & -1.398 & -0.637 \\
\hline$E_{2(j)}$ & 0.148 & 0.071 & 0.044 & 0.190 & 0.106 & 0.138 & 0.063 \\
\hline$\beta_{2(j)}$ & 0.137 & 0.149 & 0.153 & 0.130 & 0.143 & 0.138 & 0.150 \\
\hline
\end{tabular}

comparison is made by calculating the accuracy value.

When analyzed the score values, Table 14 shows that the second candidate has the maximum score compared with other candidates. In addition, this candidate has the highest score for the non-technical skill factor. The fifth candidate has the thirdhighest score for the non-technical skill factor but is the last in the candidate ranking because it has a negative score for the general technical skill. In other words, while the non-technical skills of this candidate are above average, his technical skills are not sufficient.

Table 14. Score value and accuracy value for each alternative.

\begin{tabular}{ccccccc}
\hline \multirow{2}{*}{$S_{a}$} & $S_{a}^{T O B}$ & $S_{a}^{G T B}$ & $S_{a}$ & $A_{a}^{T O B}$ & $A_{a}^{G T B}$ & $A_{a}$ \\
\hline 1 & 0.234 & 0.221 & 0.228 & 0.900 & 0.908 & 0.904 \\
2 & 0.603 & 0.360 & 0.484 & 0.921 & 0.896 & 0.908 \\
3 & 0.507 & 0.229 & 0.370 & 0.902 & 0.899 & 0.900 \\
4 & 0.195 & 0.132 & 0.165 & 0.899 & 0.918 & 0.908 \\
5 & 0.504 & -0.183 & 0.138 & 0.902 & 0.817 & 0.900 \\
\hline
\end{tabular}

\section{Conclusion}

In this research, a new approach for dealing with multi-attribute assessment in the intuitionistic fuzzy environment is proposed. In this approach, attribute values are characterized by intuitionistic fuzzy numbers and the information about weights of factors and sub-factors is not certainly known. In this approach, all information on assessments is given as linguistic expressions characterized by intuitionistic fuzzy numbers. Then, intuitionistic fuzzy weighted geometric operator and intuitionistic fuzzy entropy are used to aggregate individual opinions of evaluators and obtain the entropy weights of the factors and sub-factors, respectively. The approach first fuses all individual intuitionistic fuzzy assessments into the collective intuitionistic fuzzy assessment by using the intuitionistic fuzzy weighted geometric operator. This approach reduces losing or distorting the assessment information in the process of aggregation to the minimum, as entropy is used to convert the integrated assessment of expert groups into weights.

Owing to the increasing competition of globalization, the selection of qualified employees that is appropriate for today's conditions is one of the key factors for a company's success. Hence, the proposed method is illustrated for the concept of a Tshaped engineer which is one of the popular topics of today. The application has been carried out in an international retail company that wants to select a T-shaped engineer for the position of logistics specialist. This illustration, which is defining Tshaped engineer selection as a multi-attribute evaluation problem in the literature for the first time, shows that the novel proposed approach is consistent with the way of thinking of a human and easily applicable. In addition, the proposed approach can be used in real-life decision-making or evaluation processes in many areas.
In future research, it is aimed to extend this proposed approach to the interval-valued intuitionistic fuzzy environment. Also, it is planned to develop a $\mathrm{T}$-shaped engineer evaluation approach with separate evaluation criteria for engineering fields that require more human relations (i.e. industrial engineering, business engineering, etc.) and engineering fields requiring more technical skills (i.e. mechanical engineering, electrical-electronics engineering, computer engineering, etc.).

\subsection{Appendix}

Attributes (Skills) affiliated to sub-factors.

\begin{tabular}{ccl}
\hline SBF & Att. & Skill Expression \\
No & No & an ability to speak fluently and clean \\
\hline $1(1)$ & 1 & an \\
$1(1)$ & 2 & an ability to make presentation effectively \\
$1(1)$ & 3 & an ability to communicate effectively orally \\
$1(1)$ & 4 & an ability to use body language \\
$1(1)$ & 5 & an ability to communicate effectively in writing \\
$1(1)$ & 6 & an ability to adapt to the working environment \\
$1(1)$ & 7 & an ability to manage conflict \\
$1(1)$ & 8 & an ability to have a multidisciplinary and global \\
$1(1)$ & 9 & an ability to form good relationships with people \\
$1(1)$ & 10 & an ability to work with people from different social and \\
$1(1)$ & 11 & an ability to persuade/negotiate \\
$1(1)$ & 12 & an ability to express opinions in defining problems and \\
$1(1)$ & 13 & solutions ability to work under pressure \\
$1(1)$ & 14 & an ability to accept criticism in a polite way \\
$1(1)$ & 15 & an ability to deal with difficult people \\
$1(2)$ & 1 & an ability to function on multidisciplinary teams \\
$1(3)$ & 1 & an ability to follow innovations in the field \\
$1(3)$ & 2 & an ability to use databases and other sources of \\
$1(3)$ & 3 & anformation to conduct a literature search \\
$1(3)$ & 4 & an ability to have knowledge of contemporary issues \\
$1(3)$ & 5 & an ability to self-learning \\
$1(3)$ & 6 & an ability to have a tendency to life-long learning \\
$1(4)$ & 1 & an ability to think analytically \\
$1(4)$ & 2 & an ability to think critically \\
$1(4)$ & 3 & an ability to think creatively \\
$1(4)$ & 4 & ability to recognize what results are important \\
$1(4)$ & 5 & an ability to focus easily on the main idea required for \\
$1(4)$ & 6 & an ability to identify and analyze complex problems \\
$1(4)$ & 7 & an ability to find original alternative solutions to \\
$1(4)$ & 8 & an ability to compare and decide solution alternatives \\
$1(4)$ & 9 & an ability to follow the necessary process for the \\
& & procedure of work \\
\hline
\end{tabular}


1(5) 1 an ability to motivate, manage and supervise team members

2(1) 1 an ability to use at least one programming language effectively

2(2) 1 an ability of data collection, statistical analysis, and interpretation

2(3) 1 an ability to write effective technical reports and understand written reports

2(4) 1 an ability to use softwares, information and communication technologies related to the field

2(5) 1 an ability to identify requirements by analyzing a system, system component, or process

2(6) 1 an ability to interpret and analyze economic data

2(7) 1 an ability to use a foreign language at least at the level $\mathrm{B} 1$ of the European language portfolio

\section{References}

[1] K. T. Atanassov, "Intuitionistic fuzzy sets," Fuzzy Sets Syst, vol. 20, no. 1, pp. 87-96, Aug.1986, DOI: 10.1016/S01650114(86)80034-3.

[2] K. T. Atanassov, "More on intuitionistic fuzzy sets," Fuzzy Sets Syst, vol. 33, no.1, pp. 37-45, Oct.1989, DOI: 10.1016/0165-0114(89)90215-7.

[3] L. A. Zadeh, "Fuzzy sets," Inf.Control, vol. 8, no. 3, pp. 338-353, Jun. 1965, DOI: 10.1016/S0019-9958(65)90241-X.

[4] J. Puri and S. P. Yadav, "Intuitionistic fuzzy data envelopment analysis: An application to the banking sector in India," Expert Syst Appl, vol. 42, no. 11, pp. 4982-4998, Jul. 2015, DOI: 10.1016/j.eswa.2015.02.014.

[5] E. Szmidt and J. Kacprzyk, "Intuitionistic fuzzy sets in intelligent data analysis for medical diagnosis," in Proc. ICCS, Berlin, Heidelberg, 2018, pp. 263-271, DOI: 10.1007/3-540-45718-6_30.

[6] J. Y. Ahn, S. Y. Oh, and C. D. Lee, "An application of intervalvalued intuitionistic fuzzy sets for medical diagnosis of headache," Int J Innov Comput I, vol. 7, no. 5, pp.2755-2762, May. 2011.

[7] N. T. Thong, "HIFCF: An effective hybrid model between picture fuzzy clustering and intuitionistic fuzzy recommender systems for medical diagnosis," Expert Syst Appl, vol. 42, no. 7, pp. 36823701, May. 2015, DOI: 10.1016/j.eswa.2014.12.042.

[8] A. Kumar and M. Kaur, "A ranking approach for intuitionistic fuzzy numbers and its application," J Appl Res Technol, vol. 11, no. 3, pp. 381-396, Jun. 2013, DOI: 10.1016/S16656423(13)71548-7.

[9] F. E. Boran, S. Genç, M. Kurt, and D. Akay, "A multi-criteria intuitionistic fuzzy group decision making for supplier selection with TOPSIS method," Expert Syst Appl, vol. 36, no. 8, pp. 1136311368, Oct. 2009, DOI: 10.1016/j.eswa.2009.03.039.

[10] M. Izadikhah, "Group decision making process for supplier selection with TOPSIS method under interval-valued intuitionistic fuzzy numbers," Advances in Fuzzy Systems, vol. 2012, pp. 1-14, Nov. 2011, DOI: 10.1155/2012/407942.

[11] K. Devi and S. P. Yadav, "A multicriteria intuitionistic fuzzy group decision making for plant location selection with ELECTRE method," Int J Adv Manuf Tech, vol. 66, no. (9-12), pp. 1219-1229, Oct. 2013, DOI 10.1007/s00170-012-4400-0.

[12] Z. Yue and Y. Jia, "A group decision making model with hybrid intuitionistic fuzzy information," Comput Ind Eng, vol. 87, pp. 202-212, Sept. 2015, DOI: /10.1016/j.cie.2015.05.016.

[13] P. Gupta, M. K. Mehlawat, and N., Grover, "Intuitionistic fuzzy multi-attribute group decision-making with an application to plant location selection based on a new extended VIKOR method," Inf. Sci., vol. 370-371, pp. 184-203, Jul. 2016.

[14] Z. J. Wang and K. W. Li, "Group decision making with incomplete intuitionistic preference relations based on quadratic programming models," Comput Ind Eng, vol. 93, pp. 162-170, Mar. 2016, DOI: 10.1016/j.cie.2016.01.001

[15] I. K. Vlachos and G. D. Sergiadis, "Intuitionistic fuzzy information-applications to pattern recognition," Pattern Recognit. Lett., vol. 28, no.2, pp. 197-206, Jan. 2007, DOI: 10.1016/j.patrec.2006.07.004.

[16] F.E. Boran and D. Akay, "A biparametric similarity measure on intuitionistic fuzzy sets with applications to pattern recognition," Inf. Sci., vol. 255 , no. 10, pp. 45-57, Jan. 2014, DOI: 10.1016/j.ins.2013.08.013.

[17] F. Meng and X. Chen, "Entropy and similarity measure of Atanassov's intuitionistic fuzzy sets and their application to pattern recognition based on fuzzy measures," Pattern Anal Appl, vol. 19, no.1, pp. 11-20, Jun. 2016, DOI: DOI 10.1007/s10044-014-0378-6.

[18] D. Yu, "Prioritized information fusion method for triangular intuitionistic fuzzy set and its application to teaching quality evaluation," Int J Intell Syst, vol. 28, no. 5, pp. 411-435, Feb. 2013, DOI: doi.org/10.1002/int.21583.

[19] Z. Xu and N. Zhao, "Information fusion for intuitionistic fuzzy decision making: an overview," Inform Fusion, vol. 28, pp. 10-23, 2016, DOI: 10.1016/j.inffus.2015.07.001.

[20] A. R Mishra, D. Jain, and D. S. Hooda, "Exponential intuitionistic fuzzy information measure with assessment of service quality," Int J Fuzzy Syst, vol.19, no.3, pp. 788-798, Jan. 2017, DOI: 10.1007/s40815-016-0278-6.

[21] I. Otay, B. Oztaysi, S.C. Onar, and C. Kahraman, "Multi-expert performance evaluation of healthcare institutions using an integrated intuitionistic fuzzy AHP\&DEA methodology," KnowlBased Syst, vol. 133, pp. 90-106, Oct. 2017, DOI: 10.1016/j.knosys.2017.06.028.

[22] L. Wu, G. Wei, H. Gao, and Y. Wei, "Some interval-valued intuitionistic fuzzy dombi hamy mean operators and their application for evaluating the elderly tourism service quality in tourism destination," Mathematics, vol. 6, no.12, pp. 294, Dec. 2018, DOI: 10.3390/math6120294.

[23] Z. Xu, "Multi-person multi-attribute decision making models under intuitionistic fuzzy environment," Fuzzy Optim Decis Ma, vol. 6, no. 3, pp. 221-236, Aug. 2007b, DOI 10.1007/s10700-007-9009-7.

[24] F. E. Boran, S. Genç, and D. Akay, "Personnel selection based on intuitionistic fuzzy sets," Hum Factor Ergon Man, vol. 21, no.5, pp. 493-503, Jan. 2011, DOI: 10.1002/hfm.20252.

[25] S. Pramanik and D. Mukhopadhyaya, "Grey relational analysis based intuitionistic fuzzy multi-criteria group decision-making approach for teacher selection in higher education," Int. J. Comput. Appl., vol. 34, no.10, pp. 21-29, Oct. 2011, DOI: 10.1016/j.knosys.2017.06.028.

[26] S. F. Zhang and S. Y. Liu, "A GRA-based intuitionistic fuzzy multi-criteria group decision making method for personnel selection," Expert Syst Appl, vol. 38, no. 9, pp. 11401-11405, Sept. 2011, DOI: 10.1016/j.eswa.2011.03.012.

[27] D. Yu, W. Zhang, and Y. Xu, "Group decision making under hesitant fuzzy environment with application to personnel evaluation," Knowl-Based Syst, vol. 52, pp. 1-10, Nov. 2013, DOI: 10.1016/j.knosys.2013.04.010.

[28] O. Bali, M. Dagdeviren and S. Gumus, "An integrated dynamic intuitionistic fuzzy MADM approach for personnel promotion problem," Kybernetes, vol. 44, no. 10, pp. 1422-1436, 2015, DOI 10.1108/K-07-2014-0142.

[29] S. N. Conley, R. W. Foley, M.E. Gorman, J. Denham, and K. Coleman, "Acquisition of T-shaped expertise: an exploratory study," Soc. Epistemol., vol. 31, no. 2, pp. 165-183, Feb. 2017, DOI: $10.1080 / 02691728.2016 .1249435$.

[30] J. Tranquillo, "The T-Shaped Engineer," Journal of Engineering 
Education Transformations, vol. 30, no. 4, pp. 12-24, Apr. 2017, DOI: $10.16920 /$ jeet/2017/v30i4/114100

[31] I. F. Oskam, "T-shaped engineers for interdisciplinary innovation: an attractive perspective for young people as well as a must for innovative organizations," in Proc. 37th Annual ConferenceAttracting students in Engineering, (Vol. 14), Rotterdam, Netherlands, 2009,

[32] G. Sharma and P. Sharma, "Importance of Soft skills development in 21st century Curriculum," International Journal of Education \& Allied Sciences, vol. 2, no. 2, pp. 39-44, Jul.-Dec. 2010.

[33] H. K. Yaacoub, F. Husseini and Z. Choueiki, "Engineering soft skills: a comparative study between the GCC area demands and the ABET requirements," Competition Forum, vol. 9, no. 1, 88, 2011.

[34] F. Ahmed, L. F. Capretz, and P. Campbell, "Evaluating the demand for soft skills in software development," IT Prof, vol. 14, no. 1, pp. 44-49, Jan./Feb. 2012, DOI: 10.1109/MITP.2012.7.

[35] I. Direito, A. Pereira, and A. M. de Oliveira Duarte, "Engineering undergraduates' perceptions of soft skills: Relations with selfefficacy and learning styles," Procedia Soc Behav Sci, vol. 55, no. 5, pp. 843-851, Oct. 2012, DOI: 10.1016/j.sbspro.2012.09.571.

[36] M. M. Robles, "Executive perceptions of the top 10 soft skills needed in today's workplace," Bus. Commun. Q., vol. 75, no. 4, pp. 453-465, Oct. 2012, DOI: 10.1177/1080569912460400.

[37] A. Zaharim, I. Ahmad, Y. M. Yusoff, M. Z. Omar, and H. Basri, "Evaluating the soft skills performed by applicants of Malaysian engineers," Procedia Soc Behav Sci, vol. 60, pp. 522-528, Oct. 2012, DOI: 10.1016/j.sbspro.2012.09.417.

[38] R. Abbas, F. A. Abdul Kadir, and I. A. Ghani Azmie, "Integrating soft skills assessment through soft skills workshop program for engineering students at University of Pahang: an analysis," Int $J$ Research in Social Science, vol. 2, no.1, pp.33-46, May. 2013.

[39] A. Esa, A. Selamat, S. Padil, and J. Jamaludin, "Applications of soft skills in engineering programme at polytechnic Malaysia," Procedia Soc Behav Sci, vol.140, pp. 115-120, Aug. 2014, DOI: 10.1016/j.sbspro.2014.04.395.

[40] A. Sousa and A. Mouraz, "Promoting'Soft Skills' from the Start of the Engineering Degree and the Case Study of the Special'Projeto FEUP'Course," Int J Eng Educ., vol. 30, no.6(B), pp. 1539-1548, Jul. 2014

[41] N. Seetha, "Are soft skills important in the workplace? -A preliminary investigation in Malaysia," Int. J. Acad, vol. 4, no. 4, 44-56, Apr. 2014, DOI: 10.6007/IJARBSS/v4-i4/751.

[42] S. Naiem and M. M. Abdellatif, "Evaluation of Computer Science and Software Engineering Undergraduate's Soft Skills in Egypt from Student's Perspective," Computer and Information Science, vol. 8, no.1, pp. 36-53, Jan. 2015, DOI: 10.5539/cis.v8n1p36.

[43] E. Taylor, "Investigating the perception of stakeholders on soft skills development of students: Evidence from South Africa," Interdisciplinary Journal of e-Skills and Lifelong Learning, vol. 12, no. 1, pp.1-18, Feb. 2016, Retrieved from http://www.ijello.org/Volume12/IJELLv12p001018Taylor2494.pdf

[44] L. I. Ballesteros Sánchez, I. Ortiz Marcos, R. Rodríguez Rivero, and J. Juan Ruiz, "Project management training: An integrative approach for strengthening the soft skills of engineering students," Int J Eng Educ, vol. 33, no. 6 (A), pp. 1912-1926, Jul. 2017.

[45] F. L. Barros and R. A. Bittencourt, "Evaluating the influence of PBL on the development of soft skills in a computer engineering undergraduate program," in Proc. FIE, San Jose, California, 2018, pp. 1-9, DOI: 10.1109/FIE.2018.8658832.

[46] T.T. Wilson and A. L. Marnewick, "A comparative study of soft skills amongst the Washington accord engineering degree graduates with industry expectations," in Proc. ICETI, Stuttgart,
Germany, 2018, pp. 1-6, DOI: 10.1109/ICE.2018.8436282.

[47] H. Chaibate, A. Hadek, S. Ajana, S. Bakkali, and K. Faraj, "Analyzing the engineering soft skills required by Moroccan job market," in Proc. ICOA, Kenitra, Morocco, pp. 1-6, 2019, DOI: 10.1109/ICOA.2019.8727712.

[48] D. Ktoridou, E. Doukanari, and A. Karayiannis, "Educating the New Generation of Engineering Managers to Stay Relevant in the 21 st Century Workforce," in Proc. EDUCON, Dubai, United Arab Emirates, 2019, pp. 1547-1551, DOI: 10.1109/EDUCON.2019.8725177.

[49] R. S. Dubey and V. Tiwari, "Operationalization of soft skill attributes and determining the existing gap in novice ICT professionals," Int J Inf Manage., vol. 50, pp. 375-386, Feb. 2020, DOI: 10.1016/j.ijinfomgt.2019.09.006

[50] Z. Yue, "Deriving decision maker's weights based on distance measure for interval-valued intuitionistic fuzzy group decision making," Expert Syst Appl, vol. 38, no. 9, pp. 11665-11670, Sept. 2011, DOI: 10.1016/j.eswa.2011.03.046.

[51] J. Ye, "Multiple attribute group decision-making methods with unknown weights in intuitionistic fuzzy setting and interval-valued intuitionistic fuzzy setting," Int J Gen Syst, vol. 42, no. 5, pp. 489502, Mar. 2013, DOI: 10.1080/03081079.2013.775127.

[52] S. Liu, W. Yu, L. Liu, and Y. Hu, "Variable weights theory and its application to multi-attribute group decision making with intuitionistic fuzzy numbers on determining decision maker's weights,". PloS one, vol. 14, no. 3, pp. 1-21, Mar. 2019, DOI: 10.1371/journal.pone.0212636.

[53] S. K. De, R. Biswas, and A. R. Roy, "Some operations on intuitionistic fuzzy sets," Fuzzy Sets Syst, vol. 114, no. 3, 477-484, Sept. 2000, DOI: 10.1016/S0165-0114(98)00191-2.

[54] S. M. Chen and J. M. Tan, "Handling multicriteria fuzzy decisionmaking problems based on vague set theory," Fuzzy Sets Syst, vol. 67, no. 2, pp. 163-172, Oct. 1994, DOI: 10.1016/01650114(94)90084-1.

[55] D. H. Hong and C. H. Choi, "Multicriteria fuzzy decision-making problems based on vague set theory," Fuzzy Sets Syst, vol. 114, no. 1, pp. 103-113, Aug. 2000, DOI: 10.1016/S0165-0114(98)00271-1.

[56] Z. Xu and R. R. Yager, "Some geometric aggregation operators based on intuitionistic fuzzy sets," Int J Gen Syst, vol. 35, pp. 417433, Nov.2006, DOI: 10.1080/03081070600574353.

[57] $\mathrm{Z} . \mathrm{Xu}, \quad$ "Intuitionistic fuzzy aggregation operators," IEEE T Fuzzy Syst, vol. 15, pp. 1179-1187, Dec. 2007a, DOI: 10.1109/TFUZZ.2006.890678.

[58] T. Y. Chen and C. H. Li, "Determining objective weights with intuitionistic fuzzy entropy measures: A comparative analysis," Inf. Sci., vol. 180, no. 21, pp. 4207-4222, Nov. 2010, DOI: 10.1016/j.ins.2010.07.009.

[59] J. Z. Wu and Q. Zhang, "Multicriteria decision making method based on intuitionistic fuzzy weighted entropy," Expert Syst Appl, vol. 38, no. 1 , pp. 916-922, Jan. 2011, DOI: 10.1016/j.eswa.2010.07.073.

[60] P. Burillo and H. Bustince, "Entropy on intuitionistic fuzzy sets and on interval-valued fuzzy sets," Fuzzy Sets Syst, vol. 78, no. 3, pp. 305-316, Mar. 1996, DOI: 10.1016/0165-0114(96)84611-2.

[61] A. De Luca and S. Termini, "A Definition of non-probabilistic entropy in the setting of fuzzy sets theory," Inf.Control, vol. 20, pp. 301-312, 1972

[62] J. Ye, "Two effective measures of intuitionistic fuzzy entropy," Computing, vol. 87, no. (1-2), pp. 55-62, Jan. 2010, DOI: 10.1007/s00607-009-0075-2.

[63] I. Markes, A. Maddocks, S. Bamforth, and A. R. Crawford, "UK SPEC and the RAPID Progress File: a tool for academic, personal and professional development in engineering". in Proc. ICEE, 
Florida, USA, 2004, pp. 1-7.

[64] S. A. Male, M. B. Bush, and E. S. Chapman, E. S. "An Australian study of generic competencies required by engineers," Eur. J. Eng. Educ., vol. 36, no. 2, pp. 151-163, Jun. 2011, DOI: 10.1080/03043797.2011.569703

[65] T. Vedhathiri, "Enhancing Professional and Soft Skills of the Indian Engineering Graduates," J Eng Technol, vol. 10, no. 2, pp.117, Jul. 2016, Retrieved from http://www.ifees.net/wpcontent/uploads/2017/03/Paper-NITTTR-CH-Journal.pdf

[66] C. Andersson and D. Logofatu, "Using cultural heterogeneity to improve soft skills in engineering and computer science education," in Proc. EDUCO, Canary Islands, Spain, 2018, pp. 191-195, DOI: 10.1109/EDUCON.2018.8363227. 